Medizinhistorisches Institut der Universität Basel

Leiter: Prof.Dr.med.H.Buess

\title{
Die Anfänge der allgemeinen pathologischen Anatomie, insbesondere in der Pariser Schule
}

\section{Von Heinrich Buess}

Der große Forscher Rudolf Virchow, dessen unsterbliche Verdienste um die moderne Pathologie hier im einzelnen nicht zur Diskussion stehen, ist je und je auch als aktiver Gelehrter auf historischem Gebiet hervorgetreten. Als ein Beleg von vielen sei der richtungweisende Satz zitiert aus dem Vorwort zum ersten Band des Handbuch der speciellen Pathologie und Therapie, wo sich ein warmes Bekenntnis zur Tüchtigkeit der «alten Ärzte» findet. Virchow schreibt: «Für mich beginnt die Medicin nicht von heute, und ich halte es für unmöglich, darin ganz zu Hause zu sein, wenn man sie nicht genetisch auffaßt. So ist es gekommen, daß ich mit dem Fieber und der Entzündung, die mancher schon begraben geglaubt hatte, anfing, ganz im Sinne des grauen Altertums und der vergangenen Zeiten.»

Es dürfte aus diesen Sätzen klargeworden sein, daß ich sie nicht nur deshalb ausgewählt habe, um Virchows Liebe zur Problemgeschichte zu zeigen. Nein, vielmehr läßt schon dieser eine Satz erkennen, wie sehr gerade allgemein pathologische Begriffe hier eine wichtige Rolle spielen. Und dies, obgleich es als Nachschlagewerk der speziellen Pathologie benannt ist. Eine weitere Vertiefung schon in diesen ersten Band läßt ferner erkennen, daß wir es in den ersten drei Abschnitten wohl mit der ersten handbuchmäßigen Darstellung der allgemeinen pathologischen Anatomie im heutigen Sinn zu tun haben. Die späteren Kapitel bilden dann großenteils eine Widerlegung der Rokitanskischen Krasenlehre. An deren Stelle werden mit den Mitteln der Chemie und vor allem der Mikroskopie neue Ansätze für eine Beseitigung der alten Humoralpathologie bekanntgemacht.

Es erhebt sich nun die Frage, wo die Anfänge dieser hier bereits teilweise stabilisierten Lehren etwa über die Entzündung zu suchen sind. Bevor darauf eingegangen wird, möchte ich anhand des jüngsten deutschsprachigen Werkes der Allgemeinen Pathologie von Franz Büchner den in unserem Zusammenhang wesentlichen Gesichtspunkt herausstellen. Dem emeritierten Pathologen der Freiburger Universität geht es darum, in seinem Lehr- 
buch eine «Zusammenschau morphologischer Phänomene und Probleme» herauszuarbeiten, wobei die «Erfahrung des Morphologen» im Vordergrund stehen soll. Im Sinne der Definition von Ribbert-HamperL, daß die Pathologische Anatomie sich «mit den gestaltlichen Organveränderungen befaßt», werden auch wir unser Hauptaugenmerk auf die Abweichungen der morphologischen Struktur richten.

\section{Das lokalisatorisch-funktionelle Prinzip}

Ohne Zweifel steht fest, was wir soeben betont haben, daß es eine Allgemeine pathologische Anatomie (abgekürzt: APA) erst gibt, seitdem das funktionelle - methodisch gesehen also das experimentelle - Prinzip Eingang in die Krankheitslehre gefunden hat. Auf breitester Basis war dies erst der Fall, als man sich seit Virchow mit den Vorgängen in der Zelle zu befassen begann. Aber, fragen wir uns: Ist nicht der funktionelle Gedanke schon früher wirksam in der Geschichte der medizinischen Biologie? Tatsächlich ist dies der Fall. Und von dem historischen Moment an, wo sich diese Betrachtungsweise mit dem lokalisatorischen, am Gewebe orientierten Forschen zu einer Einheit zu verbinden begann, von da an können wir von APA sprechen. Läuft doch diese wesentlich darauf hinaus, im Körper überall vorkommende morphologische Elemente in ihren strukturellen Abweichungen zu erfassen. Um es gleich hier vorwegzunehmen: Das Studium dieser gemeinsamen anatomisch-physiologischen Aspekte, zu denen neuerdings die energetischen Vorgänge gekommen sind, war in ihren ersten Ausprägungen besonders die Domäne vitalistisch interessierter Ärzte.

Doch versuchen wir nun, die einzelnen Bausteine dieser modernen Wissenschaft fein säuberlich der Reihe nach, wenn auch nicht im Detail, so doch wenigstens stichwortartig zusammenzutragen. Es war vorhin von dem Bestreben nach Lokalisation die Rede. Immer wieder wird hier auf Morgagnis unsterbliches Werk, sein Handbuch aus dem Jahre 1761, hingewiesen. Doch steht sicher fest, daß er sich bei seiner nach Körperregionen gegliederten Darstellung methodisch nicht nur auf den Genfer Arzt ThÉoPHILE Bonet (1620-1689) stützt, dem er sich sehr verpflichtet weiß. Ohne Zweifel schwebt ihm auch die fundamentale Vorstellung der Krankheitslokalisation vor, die schon bei Galen (etwa 130-200) zu finden ist. Die «loci affecti», denen Galen eine seiner großen Monographien gewidmet hat, bringen schon im Titel zum Ausdruck, daß es, wie der Autor schreibt, eine gestörte Funktion ohne einen affizierten, also geschädigten Teil nicht gibt. 
Wie viele andere Konzeptionen dieses medizinischen Forschers und Denkers ist auch diese Erkenntnis über vielem Wust verlorengegangen. Aber auf das letzte Drittel des Zeitalters der Aufklärung spitzte sich doch von verschiedenen Seiten her die Situation in der Krankheitsforschung auf eine neue Lösung zu. Die auf diesen Gipfel konvergierenden Wege kamen erstens aus dem Lager der Physiologen, wo seit der Entdeckung des Blutkreislaufs immer mehr Experimente mit Strömungshindernissen angestellt worden waren. Sodann gewannen die Obduzenten von den Leichenzergliederungen immer mehr Klarheit über die Auswirkungen einer gestörten Hämodynamik, so z. B. bei krankhaften Veränderungen an den Herzklappen. Und drittens gelangten die Kliniker, zu denen auch führende Chirurgen wie Jean-Louis Petit (1674-1760) und John Hunter (1728-1793) gezählt werden dürfen, an Schußwunden, an Phlebitiden zu wichtigen neuen Vorstellungen über zwei so wichtige Vorgänge wie diejenigen der Entzündung und der Organisation des Gewebes in Narben. Auf diese Weise wurden von der morphologischen und physiologischen Seite die Materialien zu dem Bau zusammengetragen, auf den dann Albrecht Haller (1708-1777) gewissermaßen ein neues Stockwerk aufsetzen konnte.

Abgesehen von den erwähnten pathologisch-physiologischen Momenten, welche durch unzählige weitere Beispiele aus den Elementa physiologiae ergänzt werden könnten, hat jedoch ohne Zweifel Hallers eigenes experimentelles Euvre für die Gewinnung allgemein-pathologischer Gesichtspunkte eine große Rolle gespielt. Ich meine damit seine in Göttingen während vieler Monate mit seinen Assistenten durchgeführten Tierversuche an Muskeln und Nerven. Durch sie war eine wichtige allgemeine Tatsache eindeutig bewiesen worden, daß nämlich bestimmte Funktionen an bestimmte Substrate geknüpft sind: die Irritabilität (d.h. die Kontraktionsfähigkeit) an die Muskelfaser, die Sensibilität an das Vorhandensein von Nerven. Wenn auch mannigfache Fehlschlüsse vorkamen, so war doch damit ein allgemeines, für den ganzen lebenden Organismus gültiges lokalisatorisch-funktionelles Prinzip gewonnen. Nehmen wir noch hinzu die Hallersche Konzeption der "tela cellulosa», also des Zellgewebes, so haben wir die Voraussetzungen realer Natur zusammengetragen, auf denen ein Franzose, der dem Berner Gelehrten Haller in jeder Hinsicht kongenial war, seine eigenen Forschungen und Ideen in die Welt zu setzen vermochte.

Zum vollen Verständnis der Pionierleistungen der Pariser pathologischanatomisch-klinischen Schule ist indessen noch das klassifikatorische Moment heranzuziehen. Man könnte auch vom nosologischen Fundament spre- 
chen, das vor allem von dem Lehrer vieler Pariser Ärzte, von dem systemfreudigen Arzt-Philosophen Philippe Pinel (1745-1826) beigesteuert wurde. Seine Nosographie philosophique, die im Revolutionsjahr 1789 erschien, hat bei aller ihrer Überspitztheit in symptomatologischer Hinsicht und bei aller Mißachtung der Anatomie doch einen wesentlichen Einfluß auf seine Schüler ausgeübt. Dieser dürfte kurz gesagt im Hineintragen von naturhistorischen Denkschemata in die Biologie und in die Pathologie bestehen, wo sie in einer eigenwillig-ontologischen Fieberlehre gipfelte. Aber gerade diese letztere Tendenz liegt doch wohl dem Gedanken vom Prozeßhaften jeder Krankheit zugrunde, also der Vorstellung, daß eine Krankheit in symptomatischer Hinsicht verschiedene Stadien durchläuft, eine wichtige neue Konzeption, die wie ein roter Faden dann auch die morphologisch-klinische Forschung der Pariser Schule durchziehen sollte.

\section{Das nosologisch-histologische Prinzip}

Von dieser spekulativen Vorstellung aus mußte nur noch ein kleiner Schritt getan werden bis zu dem von der jüngeren Ärztegeneration dargelegten Gedanken, daß etwa ein Herd in der Lunge im Verlaufe der Krankheit bestimmte Wandlungen durchmacht. Es ist nun die neue, auf einer genialen Kombination beruhende Forschungsmethode, welche sowohl die morphologischen wie die funktionellen, die klassifikatorischen und genetischen Aspekte der krankhaften Veränderungen bestätigte und verallgemeinerte. Ohne weiter auf das Methodische einzugehen, sei pro memoria bloß festgehalten, daß die Begründung der Spitalmedizin, die sich im ersten Viertel des 19. Jahrhunderts in Paris vollzog, auf der Parallelität der sorgfältigen Untersuchung der Kranken und der ebenso minutiösen Obduktion der Leichen beruht. Auf knappem Raum ist es nicht möglich, einzugehen auf die einzelnen Repräsentanten der neuen Medizin, die fast ebenso revolutionär war wie der politische und gesellschaftliche Umsturz. Auch auf die Fachliteratur kann nicht genauer eingegangen werden (vgl. Bibliographie). Lassen Sie mich unter den Erforschern dieses Zeitabschnittes der Medizingeschichte nur einige Namen von Autoren der jüngsten Zeit aus dem französischen und deutschen Sprachgebiet nennen. Viel zu wenig bekannt sind die verschiedenen wegleitenden Arbeiten von Paul Delaunay (1878 bis 1959), einem bis zu seinem Tode unentwegt forschenden praktischen Arzt in der Banlieu von Paris, durch den ohne Zweifel die drei jetzt zu nennenden Verfasser wertvoller Quellenstudien entweder angeregt oder beein- 
flußt worden sind: nämlich mein 1958 verstorbener Lehrer und Vorgänger Johannes Karcher in Basel, dann vor allem unser seit vielen Jahren mit dieser Materie beschäftigter Jubilar in Zürich sowie - last but not least der Pariser Kliniker und Medizinhistoriker Charles Coury, der an unserem XIX. Internationalen Kongreß für Geschichte der Medizin in Basel zwei wichtige Vertreter der Pariser Schule in ein helleres Licht gerückt hat.

Wenn es unsere Aufgabe wäre, einen nach Persönlichkeiten orientierten Überblick über die sogenannte Pariser Schule zu geben, so würden wir uns einer verwirrenden Fülle von Fäden gegenübersehen, welche die geistigen Beziehungen zwischen den Angehörigen der fünf Altersstufen aufzeigen müßte. Grob gesprochen kann man sagen, daß jede dieser Gruppen durch einen Zeitraum von ungefähr zehn Jahren von der vorherigen getrennt ist (Geburtsjahr: etwa 1850, 1860, 1870 usw.). Was die Weltanschauung, die politische Haltung und die gesellschaftlich-berufliche Stellung dieser Männer betrifft, so wundert es uns nicht, die größten Verschiedenheiten, ja Gegensätze zu finden. Fortschrittsgläubige Jakobiner-Naturen und streng kirchliche Katholiken, Vertreter der neuen Freiheiten und reaktionäre Anhänger der Monarchie finden wir unter diesen wagemutigen Forschern vertreten. Für die bedeutendsten unter ihnen hat AcKerknecht festgestellt, daß sie niemals der Medizinischen Fakultät der altehrwürdigen Pariser Universität angehört haben. Es waren vor allem die durch die Revolution geschaffenen neuen Institutionen, aus deren Schoß viele der umwälzenden Erkenntnisse und Entdeckungen sproßten.

Darf ich jetzt kurz mitteilen, wie ich zu meinem speziellen Thema der APA gekommen bin? Auf der Suche nach einer Umschreibung der Pathologischen Anatomie stieß ich, angeleitet von einem literarischen Hinweis meines fachkundigen Kollegen in Zürich, auf einen Artikel von ThÉophILE R.H.Lænnec (1781-1826) im großen Dictionnaire des Sciences Médicales. Im zweiten Band (1812) dieses bekannten medizinischen Erzeugnisses der französischen Aufklärungs-Enzyklopädie sind zwei aufeinanderfolgende Artikel der «anatomie pathologique» gewidmet, deren Vorläufer übrigens in einer guten Bibliographie erfaßt sind.

Der Schöpfer einer neuen klinischen Medizin wider Willen, als welchen Ackerknecht jüngst Lænnec bezeichnet hat, begnügt sich in seinem Artikel nicht bloß mit einer Definition, auf die ich nicht eingehe, sondern bietet auf wenigen Seiten auch eine Systematik dieser Wissenschaft. Er schreibt (S. 51) : «Auf Grund dieser Überlegungen wird, wie ich glaube, vom jetzigen Standpunkt der pathologischen Anatomie aus der Gang für die Darlegung 
dieser Wissenschaft darin bestehen, daß wir zuerst gesamthaft die Veränderungen des Gewebes (altérations de texture) und die belebten Fremdkörper untersuchen; und nach einigen Allgemeinheiten über die Abweichungen der Ernährung (altérations de nutrition), der Form und der Stellung wird es nötig sein, sukzessive die beiden letzten Arten der organischen Veränderung in jedem funktionellen Apparat zu studieren.»

Dieses Programm, welches vom funktionell-lokalisatorischen Grundsatz ausgeht, leitet nun zum Gedanken der Klassifikation der Gewebsveränderungen über. Dieser wird in folgender Weise in die Tat umgesetzt, wobei der 31 jährige Gelehrte sich der Schwierigkeiten seines Vorhabens deutlich bewußt ist. Zu den genannten "altérations de nutrition», welche nach Lænnec die einfachsten zu sein scheinen, gehören die «hypertrophie» infolge vermehrter, und die "atrophie» infolge verminderter oder fehlender Ernährung. Handelt es sich bei diesen beiden Wortschöpfungen heute noch um grundlegende Begriffe, die dann von Virchow zytologisch begründet werden sollten, so läßt die nächste Gruppe (altérations de forme et de position) welche hauptsächlich die Luxationen und Hernien umfaßt, deutlich erkennen, daß hier - entsprechend der starken Beteiligung der Chirurgen an der Schaffung der Pathologischen Anatomie - die "pathologie externe» der heutigen französischen Klinik ihren begrifflichen Ursprung hat. Im deutschen Sprachgebiet sind diese mechanisch bedingten Störungen bekanntlich der allgemeinen und der speziellen Chirurgie zugeordnet.

Bei weitem die interessanteste Klasse «organischer Veränderungen » ist die dritte, welche, wie schon zitiert, die "geweblichen Alterationen» umfaßt. Das Gewebe kann nach Lænnec durch vier verschiedene Arten verändert werden: 1 . Lösung der Kontinuität, wie bei Wunden und Frakturen, 2. Anhäufung oder Austritt einer natürlichen Flüssigkeit (z. B. bei Anasarka), 3. durch die Entzündung und ihre Folgen, 4. durch die akzidentelle Entwicklung «eines Gewebes oder einer Materie, die vor der Krankheit nicht existierten ».

So interessant es wäre, Lænnec weiter in die Einzelheiten zu folgen, wo übrigens von der späteren Feindschaft mit Broussais noch nichts zu spüren ist, wir müssen es uns hier versagen, dieser ersten, als vielversprechend aufgefundenen Spur nachzugehen. Allgemein dürfen wir aber feststellen, daß wir es hier mit dem ersten Anfang nicht nur einer anatomisch-histologischen Klassifikation der Krankheiten, sondern mit einer Grundlegung der APA als solcher zu tun haben. Neben der Linie seiner späteren klinischen Arbeiten, die Lænnec zur Erfindung der indirekten Auskultation ge- 
führt hat, setzte der spätere Chefarzt am Hospital Necker und an der Charité auch die Linie der morphologischen Forschung fort und legte deren Ergebnisse in seinen großen Vorlesungen vor allem am «Collège de France» aus dem Jahre 1823 nieder, wo auch die APA stark berücksichtigt ist (Ackerknecht, 1964).

Nein, es stellt sich uns eine weitere Aufgabe, nämlich die Beantwortung folgender Fragen: 1. Wo hat das heutige funktionelle Denken der APA seinen Ursprung? und 2. Hat Lænnec als erster diese Klassifikation konzipiert? Denn bei diesem Kliniker, einem vorwiegend der hippokratischen Klinik verpflichteten Arzt, überwiegt doch das nosologische Prinzip. Auch verfügt er bereits über eine differenzierte Klassifikation auf nosologischer Grundlage.

Wir haben früher festgestellt, daß diesem funktionellen Denken im Methodischen das Tierexperiment entspricht. Es durfte also erwartet werden, daß bei einem Zurückverfolgen der Fußstapfen Lænnecs in Richtung der allgemeinen Physiologie der an diesem Wege errichtete Bau einer physiologisch begründeten Nosologie gefunden würde.

Tatsächlich ist die Brücke von Hallers Lebenswerk zur theoretischen, d.h. im Laboratorium und am Sektionstisch geleisteten Krankheitsforschung bei einem Arzt der dritten Dekade zu finden, d.h. bei einem Mann, der im dritten Jahrzehnt nach Convisart (1755-1821), dem Schöpfer der neuen Richtung, geboren wurde. Es ist dies der wohl allgemein bekannte Xavier Bichat (1771-1802), ein Biologe, der sich im Dienste der Wissenschaft recht eigentlich verzehrte und an den Folgen einer Lungentuberkulose starb.

Wo im einzelnen war nun die Überschneidung dieser allgemein-physiologischen Entwicklungslinie mit derjenigen der Pathologischen Anatomie bei Bichat zu finden? Wenn von diesem Pariser Arzt die Rede ist, geht man gewöhnlich von seiner Anatomie générale aus, einem fundamentalen Werk, in welchem tatsächlich die Gewebelehre vor allem im Bereich der normalen Anatomie begründet ist. Aber auch die Bedeutung der krankhaften Veränderung des Gewebes ist in dem wichtigen 7.Kapitel, nämlich in seinen Considérations générales, wenigstens grundsätzlich dargelegt.

Doch gelangt man bei einer Vertiefung in die bisher nicht eben reich dokumentierte Biographie dieses genialen, schlichten Forschers zu der Monographie, von der aus ein ganzes buntes Spektrum neuer Ansätze aufleuchtet. Es ist dies der ebenfalls im Jahre 1800 erschienene Traité des membranes, dessen zweiter Auflage von 1802 der Herausgeber Husson eine liebe- 
volle Würdigung Bichats vorangestellt hat. Mag man diesem etwa 300 Seiten umfassenden Werk die ursprünglich chirurgische Laufbahn seines Verfassers auch anmerken - Bichat war dem Chirurgen Desault tief verbunden -, so erhebt sich seine Darstellung doch zu den für jene Zeit höchsten physiologischen Untersuchungen, auf die wir nur eben im Zusammenhang mit der Pathologie jetzt kurz einzugehen haben.

Für die Lehre von den Membranen, zu denen auch die Schleimhäute im heutigen Sinne zählen, geht Bichat von Haller aus und gelangt dann vom speziellen systematischen Studium der verschiedenartigen Strukturen zu wichtigen Gesetzmäßigkeiten histologischer Natur. In jedem dieser Membransysteme werden aber auch die funktionellen Aspekte der Häute eingehend diskutiert. In den Vordergrund stellt Bichat dabei das «vaskuläre System ", d.h. die Blutversorgung, die denn auch in geschichtlicher Hinsicht am wichtigsten sind. Indem er Experimente an Hunden und Meerschweinchen zu Hilfe nimmt - auch in diesen hämodynamischen Untersuchungen besaß er an Haller ein gutes Vorbild (vgl. P. Wobmans) -, gelangt Bichat zur allgemeinen Gesetzmäßigkeit der Blutanfüllung unter normalen Bedingungen. Doch weitet er seinen Blick von den strukturellen und funktionellen Details auch auf krankhafte Zustände wie etwa die häufigen Hämorrhagien aus bestimmten Schleimhäuten, die Entzündungen usw.

Es scheint mir, daß in dieser generellen Heranziehung von Studien über den Blutkreislauf in den einzelnen Membranen ein wichtiges Gebiet der APA, nämlich dasjenige der so wichtigen Kreislaufstörungen, im Keime sogar schon histophysiologisch begründet ist. Unter besseren technischen Voraussetzungen (die Mikroskope von AMIcI sollten erst etwa zwei Jahrzehnte später eingeführt werden) und bei längerer Lebenszeit wäre Bichat sicher dazu gelangt, diese mehr angedeuteten als ausgebauten Befunde auch in pathologischer Richtung weiter zu verfolgen. Es sind gerade diese Ansätze Bichats, die schon in der Pariser Schule eine vertiefte Klarlegung erfahren haben (vgl. meine Schrift: Die Injektion, S. 3609). Damit kommen wir zur dritten und letzten grundsätzlichen Richtung, die auf unsere moderne Disziplin hingeführt hat.

\section{Das klinisch-chirurgische Prinzip}

Es wurde bereits gesagt, daß die Chirurgen einen großen Anteil an der Gründung der Pathologischen Anatomie haben. Da die Entstehung dieser Wissenschaft im 19.Jahrhundert als großenteils bekannt vorausgesetzt 
werden darf, können wir uns hier kurz fassen. Eindeutig ist es dem berühmtesten französischen Chirurgen des frühen 19.Jahrhunderts, Guillaume DupuYtren (1778-1835), der Leibchirurg von Louis XVIII. und Charles X. war, zu verdanken, daß im Jahre 1836 an der Universität Paris ein Lehrstuhl für Pathologische Anatomie errichtet wurde.

Weniger bekannt dürfte sein, daß der erste Inhaber dieses Lehrstuhls, Jean Cruveilhier (1791-1874), schon im Alter von 25 Jahren seine Dissertation dieser Wissenschaft gewidmet hat. Es dürfte selten sein, daß man mit einer Doktorarbeit gewissermaßen ein neues Fach in einer modernen Sprache literarisch begründet. Diesem zweibändigen Essai sur l'Anatomie Pathologique aus dem Jahre 1816 merkt man zwar das jugendliche Feuer seines Verfassers an. Dies gilt vor allem für seine «Klassifikation», die aufbaut auf einer unter seinem hochverehrten Lehrer Dupuytren verfaßten Dissertation von Marandel. Diese Arbeit, deren Kenntnis ich E.Ackerknecht verdanke, war schon am 31.August 1807 eingereicht worden. Sie befaßt sich mit einem im Zeitalter des Typhus, der Tuberkulose und der Diphtherie aktuellen Thema, nämlich mit den «Irritations».

Der Chirurg Marandel unternimmt hier den kühnen Versuch, zu dem er wohl durch seinen draufgängerischen Professor ermuntert worden war, eine Art von allgemein-pathologischer Einteilung der Krankheiten zu geben. Es können nach ihm sechs Klassen von Krankheiten unterschieden werden: die Irritationen (gewissermaßen eine Entzündungslehre in nuce), die Atonien, die «transformations organiques», die "dégénérations organiques», die «corps étrangers» und die «Abweichungen von der ursprünglichen Organisation und Struktur».

Man merkt dieser Einteilung stark das empirisch-praktische Schwergewicht an, das dann auch im Text immer wieder stark hervortritt. Etwa gegenüber dem wenig später unternommenen Versuch von Lænnec, der von anatomisch-nosologischen Grundlagen ausgeht, kann man von Marandels System sagen, daß es eine der ersten Klassifikationen im Hinblick auf die allgemeine Chirurgie (im Sinne der deutschen Literatur) gewesen ist.

Eine ähnliche Tendenz verrät auch das System von Cruveilhier, das eine Unzahl von Unterteilungen enthält, auf die hier einzugehen nicht möglich ist. In seiner allgemeinen Zielsetzung, die von vielen physiologischen Überlegungen ausgeht, läßt diese Klassifikation nun bereits Ansätze zur biochemischen Betrachtungsweise erkennen. Daran merkt man, daß die um jene Zeit in Paris überaus lebhaft betriebene organische Chemie ihre Auswirkungen auf das Denken der jungen Generation hatte. 
Über weite Strecken hin glaubt man, bei Cruveilhier ein Lehrbuch der Chirurgie vor sich zu haben. Wie bei Lænnec können wir auch hier den Schluß ziehen, daß in diesen Jahren des Umbruchs in der medizinischen Forschung zunächst das ganze überkommene Vermächtnis des 18.Jahrhunderts als schwerer Ballast mitgeschleppt werden mußte.

Erst unter den Auspizien der Zellenlehre erhielt dann auch die physiologisch orientierte Pathologische Anatomie methodisch und erkenntnismäßig die imponierende Vertiefung und Ausweitung. Diese ist an den Namen von Virchow geknüpft. Wir sind damit wieder an den Ausgangspunkt unseres vielleicht oft etwas verschlungenen geschichtlichen Spaziergangs zurückgekehrt. Von allen den genannten französischen Ärzten darf man sagen, daß sie einen der Leitsätze von Rousseaus Emile in die Tat umgesetzt haben, der da lautet: «Ich weiß, daß die Wahrheit in den Dingen liegt und nicht in meinem Geist, der sie beurteilt, und daß je weniger ich von mir selbst in dieses gefaßte Urteil hineinlege, um so sicherer bin ich, der Wahrheit näherzukommen» (zit. nach P.-Сн.-A. Louis). Noch heute hat der Forscher in der Medizin diese kritische Haltung gegenüber sich selbst mehr denn je nötig, um nicht auf trügerische Irrwege geführt zu werden.

\section{Bibliographie}

Ackerknecht E.H., Elisha Bartlett and the Philosophy of the Paris Clinical School, Bull. Hist. Med.1 (1950) 43-60.

- The History of the Paris Clinical School 1800-1850, Yb. Amer. Philos. Soc. 1951, 257-259.

- Broussais or a Fargotten Medical Revolution, Bull.Hist.Med.4 (1953) 320-343.

- La Médecine à Paris entre 1800 et 1850, Les Conférences du Palais de la Découverts, Série $D$, Nr. 58 (19. April 1958), Histoire des Sciences, Paris 1958.

- Die Therapie der Pariser Kliniker zwischen 1795 und 1840, Gesnerus 15 (1958) 151-163.

- Die Pariser Spitäler von 1800 als Ausgangspunkt einer neuen Medizin, Ciba Symposium 7 (1959) 98.

- Zum hundertsten Geburtstag von Virchows «Cellularpathologie», Virchows Arch.path. Anatomie 332 (1959) 1-5.

- Lænnec und sein Vorlesungsmanuskript von 1822, Gesnerus 21 (1964) 142-153.

Bichat Xavier, Recherches physiologiques sur la vie et la mort, Paris 1800.

- Traité des Membranes, Paris 1802.

- Allgemeine Anatomie, übersetzt von C. H. Pfaff, 1. und 2. Abteilung, Leipzig 1802/03.

- Pathologische Anatomie, aus dem Französischen übersetzt von A. W. Pestel, Leipzig 1827 (siehe auch unter MonteIL).

Broussais F.-J.-V., Histoire des Phlegmasies ou Inflammations chroniques, Band 1, Paris 1808. Büchner Franz, Allgemeine Pathologie, München/Berlin 1962. 
Buess Heinrich, Zur Geschichte des Emboliebegriffs bis auf Virchow, Schweiz.med.Jb. 1946.

- Die Injektion, Ciba-Z. (1946) Nr. 100.

- Theophil Bonet (1620-1689) und die grundsätzliche Bedeutung seines «Sepulchretum» in der Geschichte der Pathologischen Anatomie, Gesnerus 8 (1951) 32-52.

- Die Anfänge der pathologischen Physiologie auf dem Gebiet der Kreislaufforschung nach Albrecht Hallers «Elementa physiologiae» (1756-1760), Gesnerus 11 (1954) 121-151.

- Marksteine in der Entwicklung der Lehre von der Thrombose und Embolie, Gesnerus 12 (1955) 157-189.

- Der Einfluß Vesals auf die praktische Anatomie am Beispiel Felix Platters, Med.Mschr. 11 (1964) 502-507.

Coury Charles und Roger Rulliere, Petit et Serres, leur étude de la fièvre entéromésentrérique (1811-1813), La Presse Médicale 72 (1964) 2487-2490.

- L'identification de la fièvre "Entéromésentérique» (ou Typhoide) par Petit et Serres (1813), La Semaine des Hôpitaux 55/8 (1964) 3056-3064.

Cruveillier Jean, Essai sur l'Anatomie Pathologique, Bände 1 und 2, Paris 1816.

Delhoume Léon, L'école de Dupuytren. Jean Cruveilhier, Paris 1937.

Diepgen Paul, Rudolf Virchow und die ärztliche Praxis, Z. ärztl. Fortbildg. 7 (1940) $212 \mathrm{ff}$.

- Der Arzt Rudolf Virchow und die Medizin seiner Zeit, Z.Ver. Gesch.Berlins 2 (1943)1-12.

- Rudolf Virchow, Persönlichkeit und Werk, Das Deutsche Gesundheitswesen 25 (1946).

- Kernsprüche von Rudolf Virchow, Dtsch.med.J.17/18 (1952) 369-373.

- Die Universität von Rudolf Virchows Lebenswerk, Virchows Arch.322 (1952) 221-232.

- Die Lehre von der Entzündung, Mainzer Akad.Wiss.math.-naturw. Kl. (1953) Nr. 3.

Duclos Henri, Lannec, Paris 1932.

Foucault Michel, Naissance de la clinique, Paris 1963.

Karcher JohanN, Medizingeschichtliche Studie über die französische pathologisch-anatomisch-diagnostische Schule nach dem Jahre 1819, insbesondere über Bretonneau, Schweiz. med. Wschr. 58 (1928) 54, 73.

Kervan Roger, Lannec, Médecin Breton, Evolution de la Médecine, Hachette, o. J. (1955). Klemperer Paul, The Pathology of Morgagni and Virchow, Bull.Hist. Med.8(1958) 24-38.

- John Hunter's Contribution to Pathology, The Academy Bookmann 1960, Nr. 2, S. 6-9.

- Morbid anatomy before and after Morgagni, Bull. N.Y.Acad.Med.11 (1961) 741-760.

KrumbhaAr E. B., Pathology, New York 1937 (neue Auflage 1964).

Laennec R.-T.-H., Anatomie Pathologique, Dictionnaire des Sciences Médicales, Band 2, Paris 1812.

- Traité de l'Auscultation Médiate, et des Maladies des Poumons et du cœur, éd. Andral, Paris 1837.

Louıs P.-Ch.-A., Gastro-Entêrite, Fièvre putride, Adynamique, Ataxique, Typhoide, Band 2, Paris 1829.

- Gastro-Entérite, Band 1, Paris 1829.

Marandel, Essai sur les Irritations, Paris 1807.

Masshoff W., Das morphologische Prinzip in der allgemeinen Pathologie, Dtsch.med. Wschr. 14 (1965) 589-594.

Monteil Jean, Le Cours d'Anatomie Pathologique de Bichat: Un nouveau Manuscrit, Grenoble o. J. 
Mongagni J.B., De Sedibus, et Causis Morborum per Anatomen indagatis, Band 1, Louvain 1766.

Pagel Walter, Die Krankheitslehre der Phthise in den Phasen ihrer geschichtlichen Entwicklung, Beitr.Klin.Tbk.66 (1927) 66-98.

Petit M.A. und E. R.A.Serre, Fièvre entéro-mésentérique, Paris 1813.

VIrchow Rudolf, Handbuch der speciellen Pathologie und Therapie, Band 1, Erlangen 1854.

Waldendburg L., Die Tuberkulose, die Lungenschwindsucht und Scrofulose, Berlin 1869.

Wobmann Peter, Albrecht von Haller als Begründer der Hämodynamik, Diss. med., Basel 1965, Arch. Kreislaufforsch., im Druck.

Ziegler E., Historisches und Kritisches über die Lehre von der Entzündung, Zieglers Beitr. pathol. Anat. allg. Pathol. 12 (1892) 152-205. 